\title{
Generation of accelerating Airy and accelerating parabolic beams using phase-only patterns
}

\author{
Jeffrey A. Davis, ${ }^{1, *}$ Mark J. Mitry, ${ }^{1}$ Miguel A. Bandres,${ }^{2}$ Isaac Ruiz, ${ }^{1}$ \\ Kevin P. McAuley, ${ }^{1}$ and Don M. Cottrell ${ }^{1}$ \\ 'Department of Physics, San Diego State University, San Diego, California 92182-1233, USA \\ ${ }^{2}$ California Institute of Technology, Pasadena, California 91125, USA \\ ${ }^{*}$ Corresponding author: jdavis@ @sciences.sdsu.edu
}

Received 24 March 2009; revised 8 May 2009; accepted 16 May 2009; posted 26 May 2009 (Doc. ID 109056); published 5 June 2009

\begin{abstract}
We generate both accelerated Airy and accelerated parabolic beams using phase-only patterns encoded onto a liquid crystal display (LCD). The usual system length is $2 f$, where $f$ is the focal length of the Fourier transform lens. We develop a compact optical system having a total system length of $f$. However, the mask must now incorporate the Fresnel diffraction that is not provided by the reduced optical system length. Finally we incorporate the Fourier transform lens onto the mask. We obtain excellent experimental results with a phase-only pattern and a shorter optical system. This approach makes these beams much easier to implement. (C) 2009 Optical Society of America
\end{abstract}

OCIS codes: $\quad 050.1940,350.5500,230.6120,100.5090$.

\section{Introduction}

Accelerating Airy beams (AiB) and accelerating parabolic beams $(\mathrm{ApB})$ have been receiving attention because they remain almost diffraction-free while having a quadratic transverse shift during propagation over long distances. These beams are finite energy solutions to the paraxial wave equation in both Cartesian [1-4] and parabolic [5, 6 ] coordinates. Both the AiB beams and the ApB beams have been used for optical micromanipulation experiments $[7,8]$.

In practice, these beams are generated by forming the amplitude and phase proportional to the Fourier spectra of the desired beams in an input plane $P_{1}$. For the $\mathrm{AiB}$ beams, the phase patterns were encoded onto a phase-only liquid crystal display (LCD). The necessary Gaussian amplitude distribution was obtained by illuminating the LCD with a Gaussian beam whose diameter had been carefully prepared $[2,3]$. For the ApB beams, the amplitude distributions are much more complicated, and the authors

0003-6935/09/173170-07\$15.00/0

(C) 2009 Optical Society of America encoded both the amplitude and phase onto a single phase-only LCD [6].

In both cases, the optical Fourier transforms (producing the desired $\mathrm{AiB}$ or $\mathrm{ApB}$ beams) were formed in the focal plane $P_{2}$ of an external lens as discussed in Section 6. The transverse deflection varies as the square of the distance $z$ from this focal plane.

There are several problems with this approach. First, it is difficult and optically inefficient to encode the amplitude information. Second, the optical Fourier transform system has a rather large system length of $2 f$, where $f$ is the focal length of the Fourier transform lens. This focal length also affects the physical size and deflection of the output beam. If we reduce the distance between the LCD and the Fourier transform lens to obtain a more compact system length of $f$, the size and deflection of the beam are adversely affected.

In this work, we have three goals. First we explore the conditions where the amplitude information can be ignored, making the generation of both kinds of beams much more convenient. Second, we explore how to compensate for the effects of moving the LCD closer to the Fourier transform lens. Finally we include the Fourier transform lens on the LCD. 
We end up with a vastly more flexible and more compact optical configuration where the Fourier spectrum and Fourier transform lens are combined and encoded as a phase-only pattern. Next we briefly review the amplitude and phase characteristics of the Fourier spectrum masks for generating these beams.

\section{Fourier Spectral Masks for Generating Accelerating Beams}

The Fourier spectrum for the two-dimensional finite energy AiB beam in Cartesian coordinates $[1,2]$ is proportional to

$$
\begin{aligned}
\mathfrak{I}[\phi]\left(k_{x}, k_{y}\right) \propto & \exp \left[i x_{0}^{3}\left(k_{x}^{3} / 3+k_{y}^{3} / 3\right)\right] \\
& \times \exp \left[-a x_{0}^{2}\left(k_{x}^{2}+k_{y}^{2}\right)\right] .
\end{aligned}
$$

Here $\left(k_{x}, k_{y}\right)$ are the spatial frequency coordinates. The parameter $a$ controls the exponential aperture function that ensures the containment of the $\mathrm{AiB}$ beams. For $a \ll 1$, the accelerating beams have a quasi-diffraction-free behavior over long distances that increase as $a$ decreases. So the size of the beam increases, and the diffraction free distance increases as the parameter $a$ decreases. The parameter $x_{0}$ controls the cubic phase terms and also controls the transverse shift in both the $x$ and $y$ directions as $x, y=z^{2} / 4 k^{2} x_{0}^{3}$. Here the distance $z$ of the output plane is measured from the focal plane $P_{2}$ of the Fourier transform lens.

The Fourier spectrum of the $n$th order finite energy ApB is proportional to []ㅡ

$$
\begin{aligned}
\mathfrak{I}\left[\phi_{n}\right]\left(k_{x}, k_{y}\right) \propto & \exp \left[i \kappa ^ { 3 } \left(k_{x}^{3} / 3-a^{2} k_{x} / \kappa^{2}\right.\right. \\
& \left.\left.+k_{x} k_{y}^{2}\right)\right] \exp \left[-a \kappa^{2}\left(k_{x}^{2}\right.\right. \\
& \left.\left.+k_{y}^{2}\right)\right] \Theta_{m}\left(\sqrt{2} k_{y} \kappa\right) .
\end{aligned}
$$

This spectrum has some similarities with the AiB Fourier spectrum, including the cubic phase term and the Gaussian envelope controlled by the parameter $a$. The major difference is the quartic oscillator polynomial $\Theta_{n}\left(\sqrt{2} k_{y} \kappa\right)$ [5] that controls the amplitude and phase of the vertical spatial frequency coordinate. To distinguish these beams from the AiB beams, the parameter $x_{0}$ for the $\mathrm{AiB}$ beams is replaced by the parameter $\kappa$ for the ApB beams. This parameter $\kappa$ controls the cubic phase term and also controls the transverse shift in the $x$ direction as $x=z^{2} / 4 k^{2} \kappa^{3}$. Again the distance $z$ of the output plane is measured from the focal plane $P_{2}$ of the Fourier transform lens.

Figure 1 compares the theoretical patterns for the amplitude (top row) and phase (bottom row) of the Fourier spectra for the AiB beam (left column), and for the ApB beams for $n=0$ (middle column) and $n=6$ (right column) with $a=0.02$. In the figures, white refers to a phase of $-\pi$ radians, while black refers to a phase of $+\pi$ radians. As mentioned above, the major difference between the Fourier spectra of the ApB beams and the two-dimensional AiB

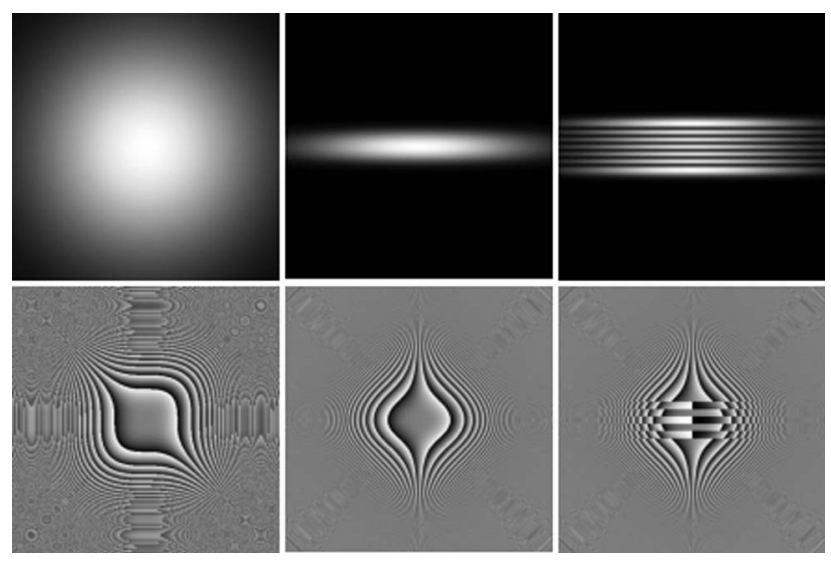

Fig. 1. Amplitude (first row) and phase (second row) Fourier spectral masks for the AiB beam (left column), and for the ApB beams for $n=0$ (middle column) and $n=6$ (right column) with $a=0.02$.

beams is the extra modulation of the amplitude and phase in the $k_{y}$ axis given by $\Theta_{n}\left(\sqrt{2} k_{y} \kappa\right)$.

\section{Experimental Results Using Amplitude and Phase Fourier Spectral Masks for Generating Accelerating Beams}

We generate experimental results for both kinds of accelerated beams by combining the amplitude and phase patterns onto a single LCD $[9,10]$. We begin with the Fourier spectrum of the desired accelerating beam function having an amplitude $M_{P}(x, y)$ and phase $\phi_{P}(x, y)$ and written as $M_{P}(x, y) \exp \left\{i\left[\phi_{P}\right.\right.$ $(x, y)]\}$. In our approach, we add a vertically oriented linear phase grating $\phi_{G}(y)=2 \pi y / d$ with period $d$ to obtain a new phase pattern as $\phi_{P}(x, y)+\phi_{G}(y)$. The amplitude information is then encoded by spatially modulating the phase pattern with the amplitude $[\underline{9}, 10]$ as $\exp \left\{i M_{P}^{\prime}(x, y)\left[\phi_{P}(x, y)+\phi_{G}(y)\right]\right\}$. The intensity that is diffracted into the first order varies spatially and reproduces the desired amplitude and phase distribution. There is a slight distortion that is corrected by modifying the amplitude term $M_{P}^{\prime}(x, y)$. Here the total phase $\phi=\left[\phi_{P}(x, y)+\right.$ $\left.\phi_{G}(x, y)\right]$ is defined in the range $[-\pi, \pi]$, while the amplitude is defined in the range $\left[0 \leq M_{P}^{\prime}(x, y) \leq 1\right]$. The zero order diffraction produces an undesired term that can be blocked.

For the experiments, linearly polarized light from an argon laser is spatially filtered, expanded, and collimated. The amplitude and phase patterns are encoded onto a parallel-aligned LCD manufactured by Seiko Epson with $640 \times 480$ pixels with pixel spacing of $42 \mu \mathrm{m}$ [11]. Each pixel acts as an electrically controllable phase plate, where the total phase shift exceeds $2 \pi$ radians as a function of gray level at the argon laser wavelength of $514.5 \mathrm{~nm}$. The linear phase grating period $d$ was 8 pixels, $\sim 328 \mu \mathrm{m}$. The Fourier transform of the mask was formed in the focal plane of a $1-\mathrm{m}$ focal length lens to create the accelerated beams. The output was then recorded with a CCD camera. To magnify the image, we mounted a $10 \times$ microscope objective at a fixed distance on the front 
of the CCD camera [6]. By translating this assembly, we recorded the propagation dynamics of the beams.

Figure 2 shows experimental results for the $\mathrm{AiB}$ beam and for ApB beams having $n=0$ and $n=6$, where $a=0.02$, and $\kappa=x_{0}=68.5 \mu \mathrm{m}$ at propagation distances of $z=\{0,15,30,45\} \mathrm{cm}$ measured from the focal plane $P_{2}$ of the Fourier transform lens. The AiB beams are characterized by an inverted L-shaped series of spots, while the $n$th order AiB beams are distinguished by $n+1$ parabolas. The width of the image plane corresponds to $4 \mathrm{~mm}$. As expected, the beams remain almost diffraction-free for $z \approx 45 \mathrm{~cm}$. Note that the "wings" of the ApB beams flare toward the center; this phenomenon is most easily seen for the $n=6$ pattern at the longer propagation distances, and this will be commented on later. The AiB beam shows a quadratic shift in the $45^{\circ}$ direction, while the ApB beams have a transverse quadratic right shift during propagation. However, by a rotation of the pattern, we can always choose an arbitrary direction for the shift. The direction of the transverse shift can be reversed using the complex conjugate of the phase mask. The results agree with previous experimental results and with theory.

\section{Experimental Results Using Phase-Only Fourier Spectral Masks for Generating Accelerating Beams}

Next, we repeated the same experiment using phaseonly patterns where the amplitude information is not
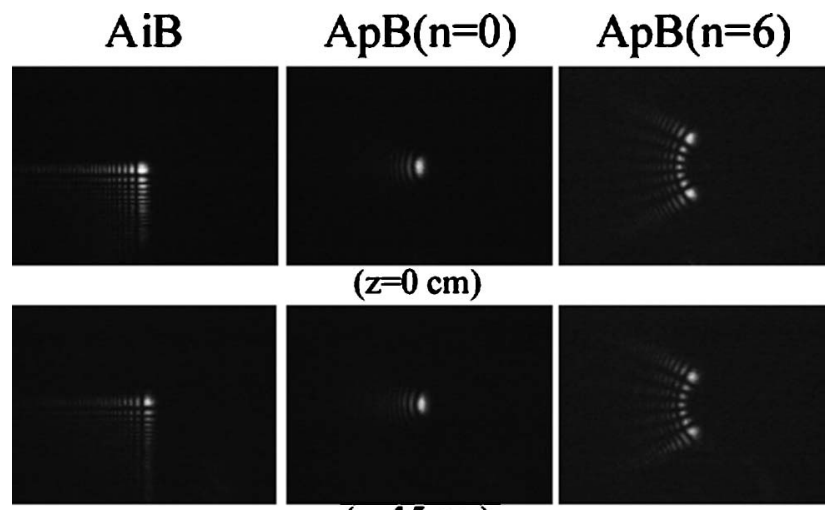

$(\mathrm{z}=15 \mathrm{~cm})$
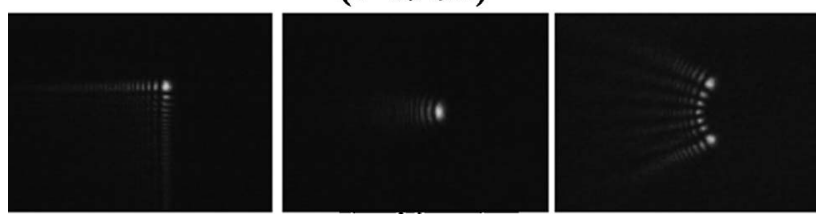

$(\mathrm{z}=30 \mathrm{~cm})$
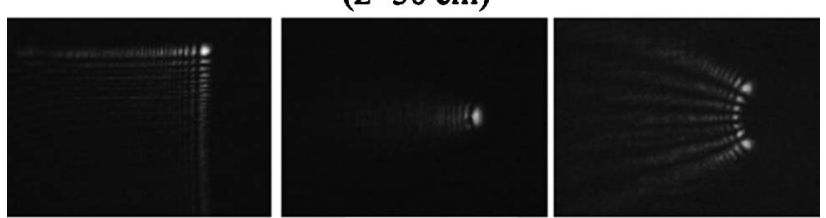

$(z=45 \mathrm{~cm})$

Fig. 2. Experimental results for $\mathrm{AiB}$ and for $\mathrm{ApB}$ beams with $n=$ 0 and $n=6$ at distances of $z=\{0,15,30,45\} \mathrm{cm}$ using amplitude and phase masks. included. Experimental results are shown in Fig. 3 , using the same experimental parameters as in Fig. $\overline{2}$.

The AiB beam results are excellent, indicating that the amplitude information is not necessary. This is an important observation and should make the generation of the AiB beams more convenient. However, the results for the ApB beams are poor, and this means that the amplitude information is critical. The $n=0$ results are identical to the AiB beam, with a $45^{\circ}$ rotation of the axes. This is expected upon examination of the phase patterns in Fig. 1 or Eqs. (1) and (2). The quality of the $n=6 \mathrm{ApB}$ beam is quite poor, and the "wings" of the beams flare away from the origin rather than toward the origin, as shown in Fig. 2.

To overcome this difficulty with the ApB beam masks, we tried encoding a horizontal binary amplitude slit on top of the phase pattern. This slit was located at the center of the phase pattern, and its vertical height could be changed. As we decreased the slit height from 120 pixels to 20 pixels, the resulting beam improves as shown in Fig. 4 for the $n=0 \mathrm{ApB}$ beam. The optimal results are obtained when the slit height is about 60 pixels. This corresponds to the $1 / e$ width of the quartic oscillator function. This characteristic seems generally appropriate for all of the cases that we considered (where $n=0,3,6$ ). The optimal results for our ApB beams were obtained when the slit height corresponded to the $1 / e$ height of the quartic oscillator function.
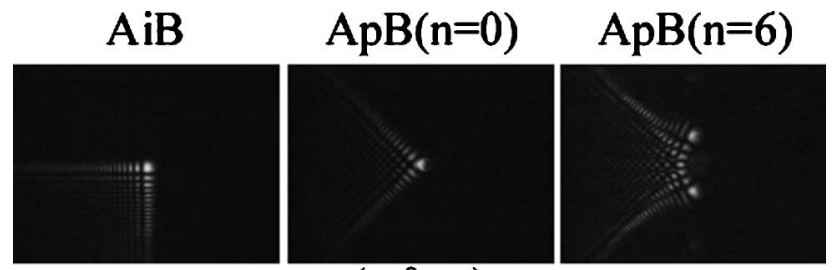

$(\mathrm{z}=0 \mathrm{~cm})$
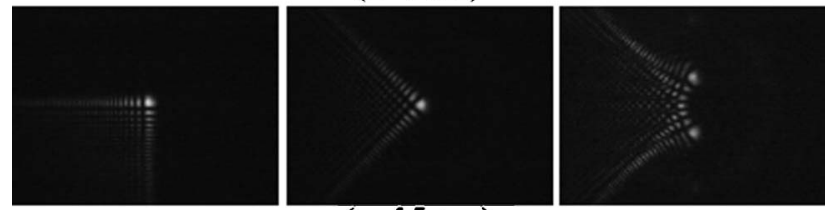

$(\mathrm{z}=15 \mathrm{~cm})$
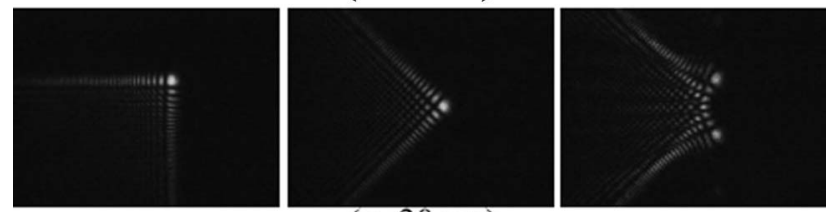

$(\mathrm{z}=30 \mathrm{~cm})$
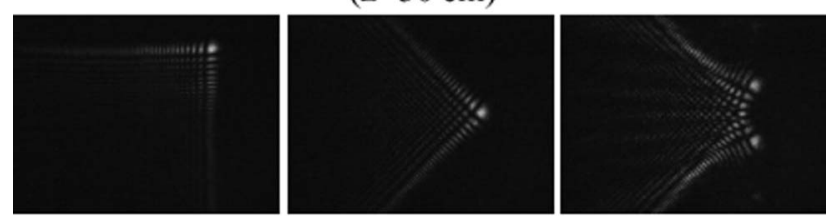

$(z=45 \mathrm{~cm})$

Fig. 3. Experimental results for $\mathrm{AiB}$ and for $\mathrm{ApB}$ beams with $n=$ 0 and $n=6$ at distances of $z=\{0,15,30,45\} \mathrm{cm}$ using phase-only masks. 


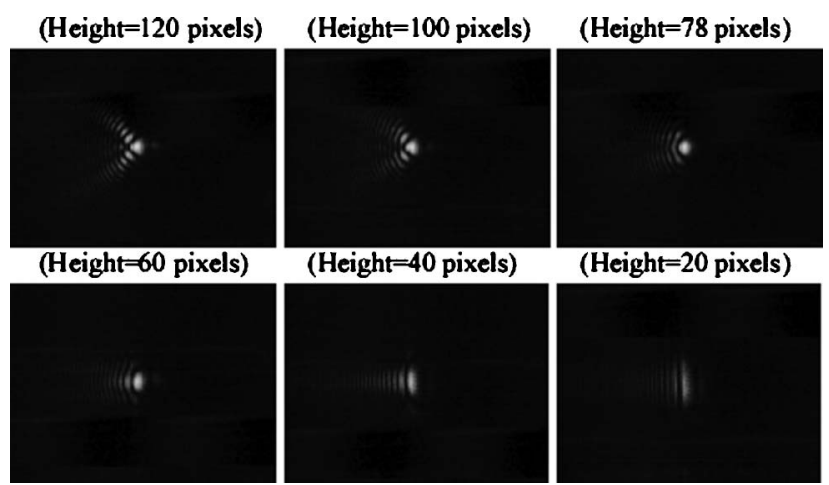

Fig. 4. Experimental results at a fixed propagation distance for ApB beam with $n=0$ using phase-only mask with different binary vertical slit widths.

Figure 5 shows the results using the modified phase-only spectral masks for the cases of the $\mathrm{AiB}$ beam, and the ApB beams for $n=0$ and $n=6$ as a function of distance. In these cases, the results agree well with the amplitude and phase results of Fig. 2. Next we examined the consequences of shortening the length of the optical Fourier system.

\section{Experimental Results Using Amplitude and Phase Fourier Spectral Masks and a Compact Optical System for Generating Accelerating Beams}

As mentioned in the introduction, the Fourier transform of the generating pattern is formed using a $2 f$ optical Fourier system, as shown in Fig. 6(a). The input plane is defined as plane $P_{1}$. The Fresnel diffrac-
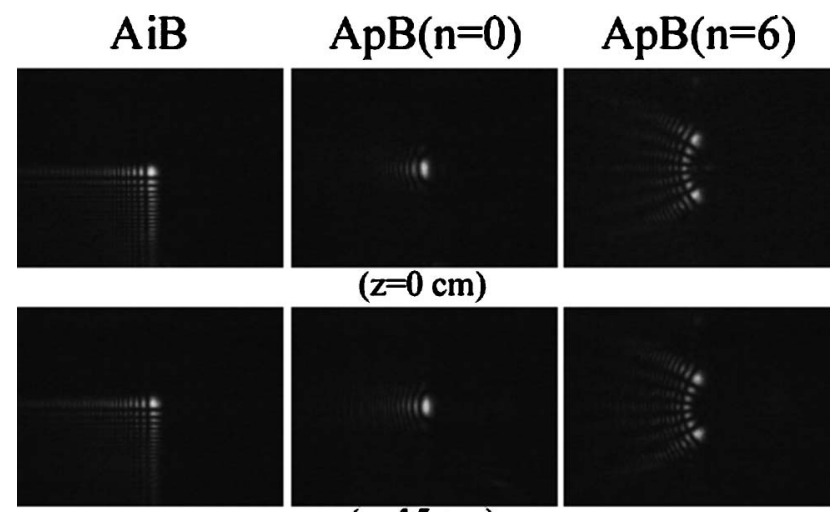

$(\mathrm{z}=15 \mathrm{~cm})$
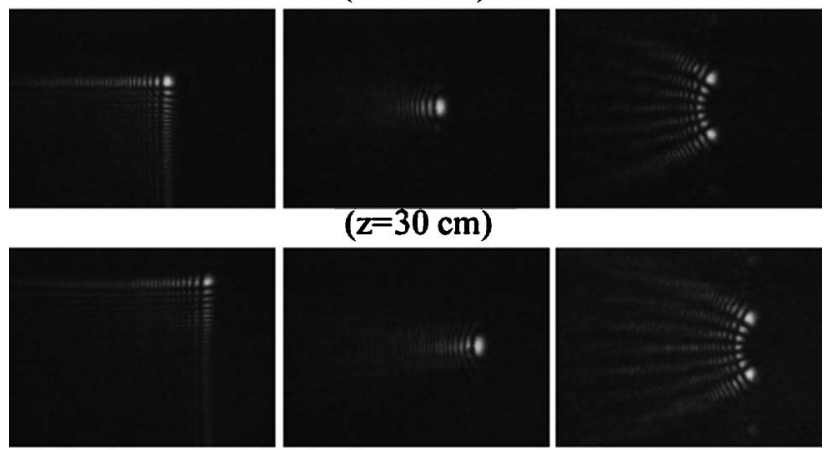

$(z=45 \mathrm{~cm})$

Fig. 5. Experimental results for AiB and for ApB beams with $n=$ 0 and $n=6$ at distances of $z=\{0,15,30,45\} \mathrm{cm}$ using phase masks with properly chosen binary slit width. tion pattern of the input is formed at a distance $d_{1}=f$, where $f$ is the focal length of the Fourier transform lens. This Fresnel diffraction pattern is multiplied by the phase function of the Fourier transform lens, and the Fresnel diffraction pattern of this product is formed in the focal plane $P_{2}$ of the Fourier transform lens at a distance $d_{2}=f$. The measurements of Fig. 2 were taken at the output plane located at various distances $z$ from the focal plane. However, the total system length is $2 f$ and can be uncomfortably long for most laboratories. Next we investigated the consequences of reducing the distance to $d_{1}=0$, forming a shortened system as shown in Fig. 6(b). This simplification is common in Fourier optical systems. We again measured the beam at various distances $z$ from the focal plane.

Figure 7 shows experimental results for the shortened system of Fig. 6(b) for the AiB beam and for ApB beams having $n=0$ and $n=6$, where $a=0.02$, and $\kappa=x_{0}=68.5 \mu \mathrm{m}$ at propagation distances of $z=\{0,15,30,45\} \mathrm{cm}$. We see two consequences. First, the sizes of the beams increase with propagation distance. This is most clearly seen for the $n=6$ case. Secondly the deflection decreases relative to the results from Fig. 2. In general, we find that the size of the beam increases and the deflection decreases as the distance $d_{1}$ decreases. These results can be understood using the ABCD optical system discussed in Ref. [4]. This theoretical approach predicts that the deflection will vary approximately as

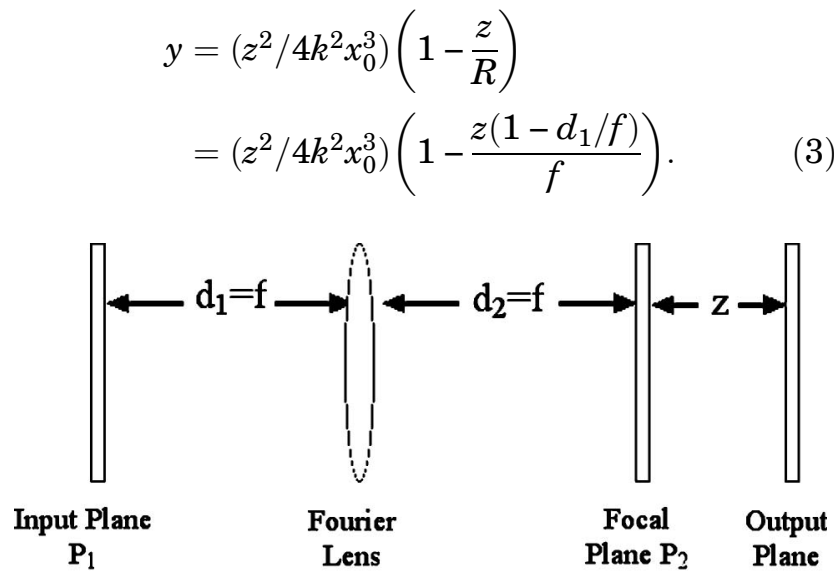

(a)

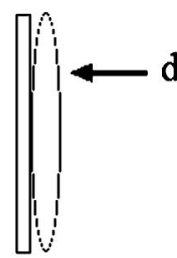

Input Plane $\mathbf{P}_{1}$

+ Fourier Lens

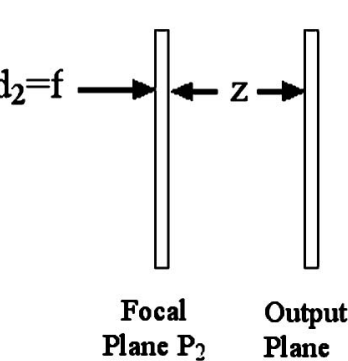

(b)

Fig. 6. (a) Conventional optical Fourier system; (b) shortened optical Fourier system. 

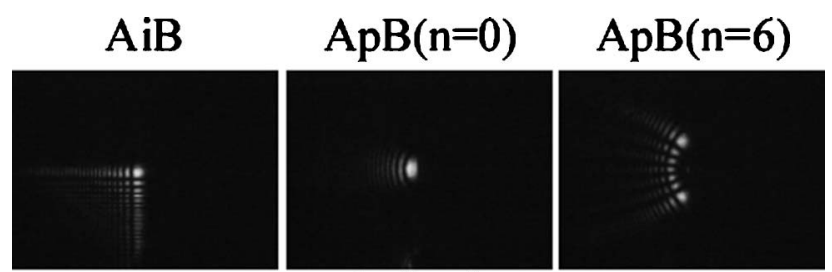

$(\mathrm{z}=0 \mathrm{~cm})$
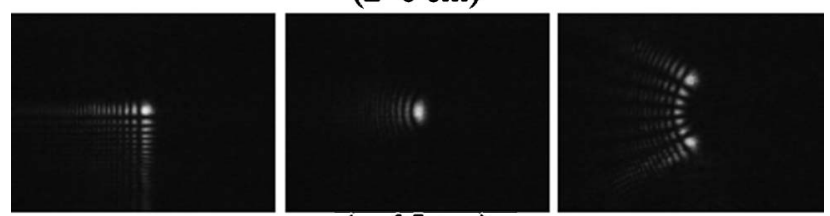

$(z=15 \mathrm{~cm})$
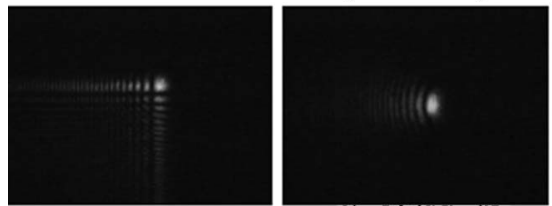

$(z=30 \mathrm{~cm})$
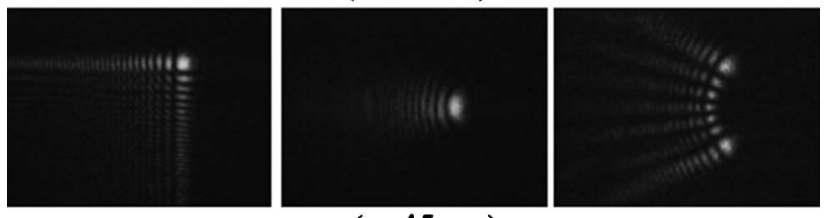

$(z=45 \mathrm{~cm})$

Fig. 7. Experimental results for AiB and for ApB beams with $n=$ 0 and $n=6$ at distances of $z=\{0,15,30,45\} \mathrm{cm}$ using amplitude and phase masks and with a shortened optical system.

Our experimental results and computer simulations are in good agreement with these predictions. Next we discuss how to retain the shorter optical system and produce excellent results with phase-only patterns.

\section{Experimental Results Using Phase-Only Fourier Spectral Masks and a Compact Optical System for Generating Accelerating Beams}

The poor performance of the shortened optical system of Fig. 6(b) can be attributed to the absence of the Fresnel diffraction over the distance $d_{1}$ between the input plane and the lens plane. We decided to implement this Fresnel diffraction into our mask function as shown in Fig. 8. Figures 8(a) and 8(b) show the amplitude and phase of the Fourier transform mask for the $n=6 \mathrm{ApB}$ beam. We then computed the Fresnel diffraction pattern formed from this input at a distance of the focal length of the lens, and the results are shown in Figs. 8(c) and 8(d). As before, white refers to a phase of $-\pi$ radians, while black refers to a phase of $+\pi$ radians. It is obvious that the amplitude and phase are both affected by this Fresnel diffraction. We encoded only the phase portion of this mask onto the LCD and examined the output at distances from the focal plane of the lens. Note that in this configuration, we did not use the horizontal slits that were required in Section $\underline{4}$.

Experimental results in Fig 9 show that the sizes, shapes, and deflections of the $\mathrm{AiB}$ and $\mathrm{ApB}$ beams are in excellent agreement with the original results
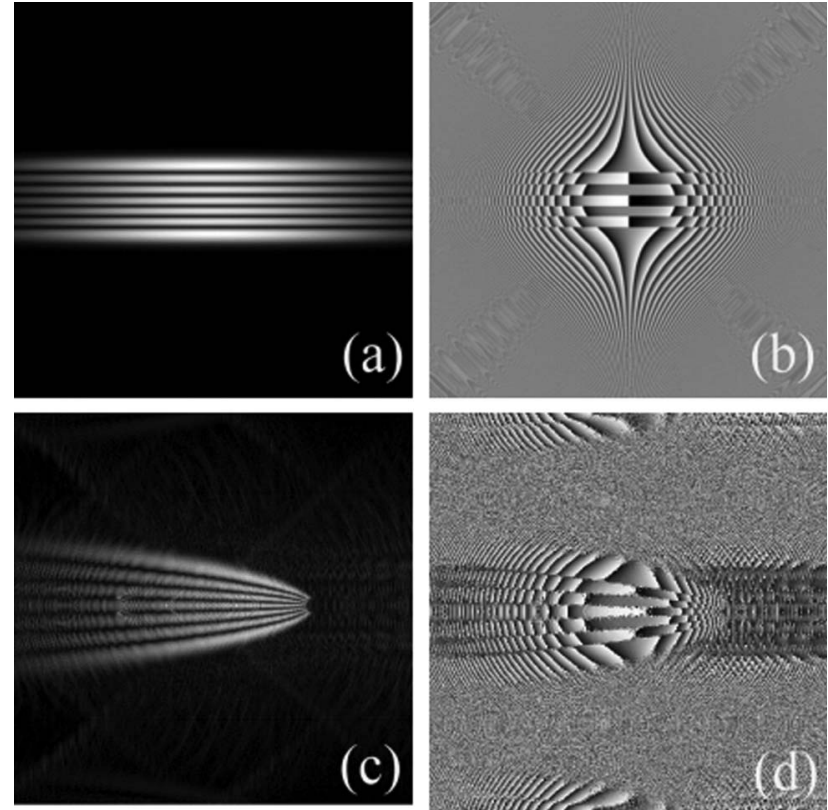

Fig. 8. Amplitude (a) and phase (b) spectral masks for ApB beam with $n=6$. Amplitude (c) and phase (d) spectral masks incorporating Fresnel diffraction through a distance of $1 \mathrm{~m}$ for an ApB beam with $n=6$.

shown in Fig. 2. However, there are two benefits to this new design; we are encoding only the phase of the Fourier spectral function, and we have a shortened optical system.
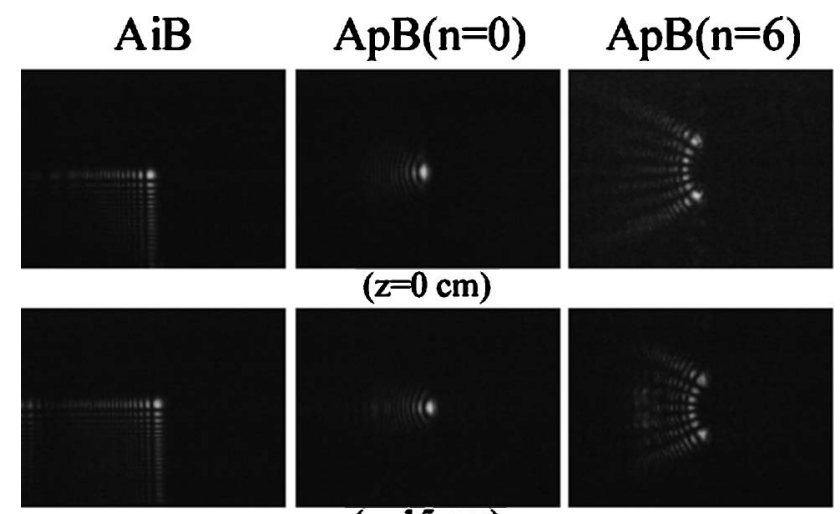

$(\mathrm{z}=15 \mathrm{~cm})$
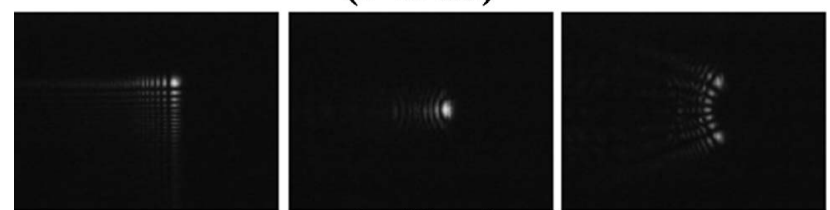

$(z=30 \mathrm{~cm})$
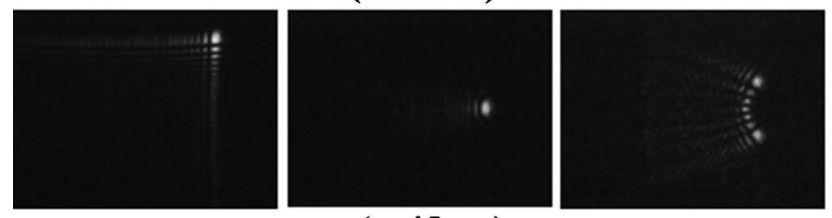

$(z=45 \mathrm{~cm})$

Fig. 9. Experimental results for $\mathrm{AiB}$ and for $\mathrm{ApB}$ beams with $n=$ 0 and $n=6$ at distances of $z=\{0,15,30,45\} \mathrm{cm}$ using phase-only masks that encode both the generating spectral mask and the effects of Fresnel diffraction with the shorter optical system. 


\section{Encoding the Fourier Transform Lens onto the Phase-Only Spectral Masks}

As a final simplification, we encoded the Fourier transform lens from Fig. 6(b) onto the phase-only spectral masks. This produces an extremely simple system, where everything (including the Fourier spectral mask, the Fresnel diffraction, and the Fourier transform lens) can be encoded onto a single programmable optical element. However, there are restrictions on the focal length that can be successfully encoded due to the pixel structure of the LCD [12]. As the focal length of the Fresnel lens decreases, the distance between the phase maxima decreases. However, the period of the oscillations in the phase function cannot be smaller than the pixel spacing. This leads to an effective Nyquist focal length limit given by $f_{N}=N \Delta^{2} / \lambda$, where $N$ is the number of pixels on the LCD and $\Delta$ is the pixel spacing. For the LCD used in these studies, the Nyquist focal length is $1.76 \mathrm{~m}$. For values smaller than this, we find decreased optical efficiency and some distortion in the encoding capabilities.

Experimental results are shown in Fig. 10 using the same conditions as before. Note that we used a focal length shorter than $f_{N}$ of $1 \mathrm{~m}$ so that the data would agree with previous results. These results in Fig 10 show that the sizes, shapes, and deflections
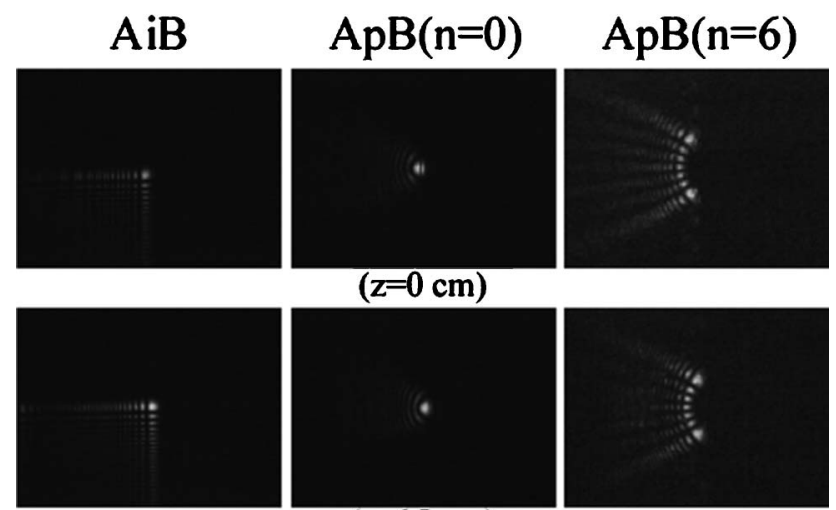

$(\mathrm{z}=15 \mathrm{~cm})$
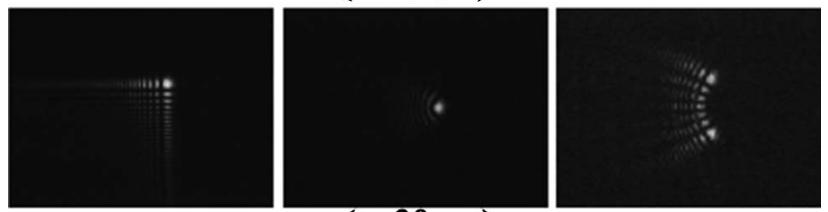

$(z=30 \mathrm{~cm})$
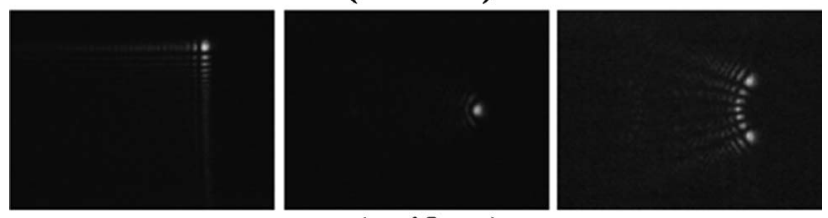

$(z=45 \mathrm{~cm})$

Fig. 10. Experimental results for $\mathrm{AiB}$ and for $\mathrm{ApB}$ beams with $n=0$ and $n=6$ at distances of $z=\{0,15,30,45\} \mathrm{cm}$ using phase-only masks that encode the generating spectral mask, the effects of Fresnel diffraction, and the Fourier transform lens, with the shorter optical system. of the $\mathrm{AiB}$ and $\mathrm{ApB}$ beams are also in excellent agreement with the original results shown in Fig. 2. As discussed earlier, the advantage of this system is that everything, including the initial phase pattern, the Fresnel diffraction, and the Fourier transform lens, is encoded onto the LCD. In addition, we obtain a shortened optical system. However, there is a problem in that the focal length of the lens can be longer than desired. This can be remedied using LCDs with smaller pixel sizes.

\section{Conclusions}

In conclusion, we have experimentally demonstrated several approaches that allow the generation of $\mathrm{AiB}$ and ApB beams using only the phase portion of the Fourier spectral generating pattern. We find that the AiB beams are very robust, while the ApB beams require more care with the amplitude information. Excellent results were obtained where we masked part of the phase mask with a slit function. We then considered a compact optical system with half of the physical length that is normally required. However, this does not yield acceptable results. We then studied a phase-only mask that combines the Fresnel diffraction corrections for the shortened optical system. Excellent experimental results are obtained for both the AiB and ApB beams. Finally we incorporated the Fourier transform lens onto the pattern and again obtained excellent output patterns. We expect that these results will be very useful for the optical community in implementing these kinds of new beams.

We would like to thank Tomio Sonehara of Seiko Epson Corporation for the use of the LCD.

\section{References}

1. G. A. Siviloglou and D. N. Christodoulides, "Accelerating finite energy Airy beams," Opt. Lett. 32, 979-981 (2007).

2. G. A. Siviloglou, J. Broky, A. Dogariu, and D. N. Christodoulides, "Observation of accelerating Airy beams," Phys. Rev. Lett. 99, 213901 (2007).

3. G. A. Siviloglou, J. Broky, A. Dogariu, and D. N. Christodoulides, "Ballistic dynamics of Airy beams," Opt. Lett. 33, 207-209 (2008).

4. M. A. Bandres and J. C. Gutiérrez-Vega, "Airy-Gauss beams and their transformation by paraxial optical systems," Opt. Express 15, 16719-16728 (2007).

5. M. A. Bandres, "Accelerating parabolic beams," Opt. Lett. 33, 1678-1680 (2008).

6. J. A. Davis, M. J. Mitry, M. A. Bandres, and D. M. Cottrell, "Observation of accelerating parabolic beams," Opt. Express 16, 12866-12871 (2008).

7. J. Baumgartl, M. Mazilu, and K. Dholakia, "Optically mediated particle clearing using Airy wavepackets," Nat. Photon. 2, 675-678 (2008).

8. J. Baumgartl, G. M. Hannappel, D. J. Stevenson, D. Day, M. Gu, and K. Dholakia, "Optical redistribution of microparticles and cells between microwells," Lab Chip 9, 1334-1336 (2009).

9. J. A. Davis, D. M. Cottrell, J. Campos, M. J. Yzuel, and I. Moreno, "Encoding amplitude information onto phase-only filters,” Appl. Opt. 38, 5004-5013 (1999). 
10. J. B. Bentley, J. A. Davis, M. A. Bandres, and J. C. Gutiérrez-Vega, "Generation of helical Ince-Gaussian beams with a liquid-crystal display," Opt. Lett. 31, 649-651 (2006).

11. J. A. Davis, P. Tsai, D. M. Cottrell, T. Sonehara, and J. Amako, "Transmission variations in liquid crystal spatial light modu- lators caused by interference and diffraction effects," Opt. Eng. 38, 1051-1057 (1999).

12. D. M. Cottrell, J. A. Davis, T. R. Hedman, and R. A. Lilly, "Multiple imaging phase-encoded optical elements written on programmable spatial light modulators," Appl. Opt. 29, 2505-2509 (1990). 